\title{
Advanced Authentication Scheme using Multimodal Biometric Scheme
}

\author{
Shreya Mohan \\ Karunya University \\ Coimbatore, India
}

\author{
Ephin M \\ Karunya University \\ Coimbatore, India
}

\begin{abstract}
Fingerprint recognition has attracted various researchers and achieved great success. But, fingerprint alone may not be able to meet the increasing demand of high accuracy in today's biometric system. The purpose of our paper is to inspect whether the integration of palmprint and fingerprint biometric can achieve performance that may not be possible using a single biometric technology. Pre-processing is done for fingerprint and palmprint images separately in order to remove any noise. The next step is feature extraction. Minutiae algorithm is used for fingerprint feature extraction and Local Binary pattern for palmprint. Wavelet fusion is applied in order to fuse the extracted features and Support Vector Machine is used for matching. The main highlight of the project is multimodal biometrics which will give a better security and accuracy comparing to unimodel system.
\end{abstract}

Keywords: fingerprint; palmprint; multimodal biometrics; minutiae; Local Binary Pattern.

\section{INTRODUCTION}

All The rapid growth in the use of Internet applications and the great concern of security require reliable and automatic personal identification. Traditional automatic personal identification schemes can be divided into two categories: knowledge-based approach, such as a password and tokenbased approach, such as an ID card, physical key and a passport. However, these approaches have limitations. In the knowledge-based approach, there are chances that, the "knowledge" can be guessed, forgotten or shared. In the token-based approach, there are chances that the "token" can be stolen or lost. These figures strongly indicate that we need a more effective and reliable solution for human identity management. Biometrics is regarded as the potential solution.

Biometric authentication refers to the technology for personal identification or authentication based on our physiological and/or behavioural characteristics. Biometrics is mainly concerned with 'what you are' rather than 'what you carry'. Biometrics is mainly used in computer science as a form of identification and access control. Biometrics is very essential in today's world in order to protect sensitive areas where public interaction is more, like railway stations and airports. Applications of biometrics can be found in many areas including electronic commerce, electronic banking and many security applications. Various methods are available and are based on different personal characteristics. Human characteristics proposed as biometric traits have both advantages and disadvantages. The selection of biometric traits depends on requirements of applications. Retina, hand geometry, iris, voice, fingerprint, face are some of the commonly used biometric traits.

Existing biometric modalities including palmprint, fingerprint, face, voice, signature etc. is now widely used in many security applications. Each one has its individual merits and demerits. Five objectives, cost, accuracy, user acceptance and environment constraints, computation speed and security should be considered when designing a biometric system. Each of the above parameters are inter-related. Reducing accuracy can increase speed. Reducing user acceptance can improve accuracy. Increasing cost can enhance security. A practical biometric system should balance all the five objectives.

Based on the literature survey, the hand-based biometric acquisition has many advantages. It has higher user acceptance and is more user friendly. The fingerprint, face, iris, palmprint modalities have been highly explored, and are nowadays available in real-world practice. Among the hand based biometric system, fingerprint identification is the oldest and the most popular one. It is the method of identification using the impressions made by the minute ridge formats or patterns found on the fingertips. The ridge patterns will be different throughout the life for every individual. Fingerprints will offer an infallible means of personal authentication. Other personal characteristics may change, but fingerprints do not. A lot of study has been carried on based on iris, finger, face etc. But the developments under palmprint is comparatively less. The inner surface of the palm normally contains distinct features like principle lines, wrinkles and ridges. The principle lines and wrinkles are formed between the third and fifth months of pregnancy and superficial lines appear after we born. Even identical twins have different palm prints. Fingerprint is a popular biometric identification technology and studies are still going on in the palmprint identification. In order to increase the performance of the automated system, it is advisable to go for multimodal biometrics. A biometric system which uses information from different biometrics is termed as multimodel biometrics. Eg: fingerprint and palmprint, iris, palmprint and face etc. Multimodal biometric techniques have attracted much attention as the add-on information between different modalities could improve the recognition performance.

A typical multimodal biometric authentication system consists of five parts. Image capture, pre-processing, feature extraction, fusion and matching. Special biometric scanners are used for image capturing. It may vary depending on the type of biometric traits used [1,2]. At the pre-processing stage the image is enhanced to remove noise and unwanted areas. Feature extraction gets effective features from the preprocessed biometric trait. Feature extraction for palmprint and fingerprint are different. After feature extraction fusion is carried out to combine different features and stored in the database as templates. A matching algorithm is used to compare it with the stored one in the database. Fig 1 gives the 
basic block diagram of a biometric system. Attention should have to be paid in the security of biometric trait also.

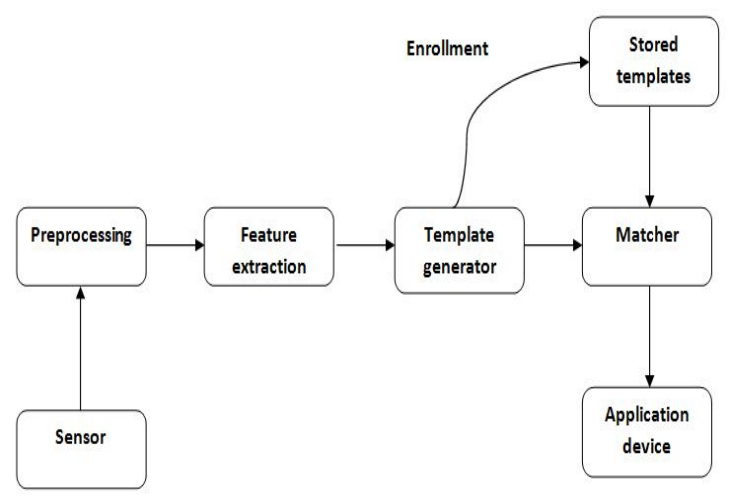

Figure 1. Basic Biometric System

Before performing multi-biometrics based on palmprint and fingerprint, it is important to understand the background for mono-modal biometrics. Various studies have shown how the palmprint and fingerprint are viable biometric features. Due to the availability of different features, different methods of evaluation, and different methods of combining the evaluations, here we will analysis some commonly available biometric methods.

\section{RELATED WORKS}

One of the initial step called pre-processing is carried out for palm print and fingerprint separately. After the image is captured by the scanner it may be distorted or blurred due to the bad environmental conditions. In these conditions a good pre-processing method is a must. Low pass filters like Gaussian can be used for smoothening. In [32] in addition to Gaussian filter, Short Time Fourier Transform (STFT) analysis is adopted to enhance fingerprint image quality. Sometimes the binarized fingerprint image contains a number of false minutiae. In [27] a detailed pre-processing is mentioned to remove false minutiae has been described.

For palmprint images, the central part, called the Region of Interest should have to be extracted. For extracting the central part, a coordination system should have to be established. There are several implementations including tangent [1], bisector $[6,7]$ and finger based $[8,9]$ to detect the key points between fingers.

After computing the coordinate systems, the central parts of palmprints, which is called the Region of Interest (ROI) are segmented. In most of the pre-processing algorithms, ROI will have square shape but some of them will have circular [10] and half elliptical shapes [11].

For extracting the features from the fingerprint image, a popular method is minutiae extraction. A fingerprint is made of a series of ridges and furrows on the surface of the finger. Minutiae extraction algorithm will find out the minute points from the fingerprint and then map their relative placement on the finger. When the fingerprint is of low quality, it will be difficult to extract the minutiae points. For that only we are using different filters and other image enhancement techniques at the pre-processing stage. The output of this algorithm will be the image template containing the minutiae details. There are two types of minutiae points. Ridge ending and Ridge bifurcation[4]. In [26] an advanced fingerprint feature extraction method is introduced through which minutiae are extracted directly from original gray-level fingerprint images without binarization and thinning. Gabor filter bank can also be used to extract features from fingerprint [24].

Comparing to fingerprint, palmprint contains more features. So a good method should be applied in order to extract all the features. The feature extraction methods can be divided into three types. Line based approach, Subspace based approach and Statistical approach. In line based approaches, they use existing edge detection methods to extract palm lines[12]. Subspace-based approaches also called appearance-based approach. They use Principal Component Analysis (PCA) [20,16], Linear Discriminant Analysis (LDA) [25] and Independent Component Analysis (ICA) [28]. Paper [21] proposes Matrix-based Complex PCA (MCPCA), that uses a complex matrix to denote two biometric traits from one subject.

Statistical approaches are two types. Local and global statistical approach. Local statistical approaches transform images into another domain and then divide the transformed images into several small regions[13,14]. Gabor, wavelets and Fourier transforms have been applied. Global statistical approaches [15] compute global statistical features directly from the whole transformed images. Moments, center of gravity and density have been regarded as the global statistical features. An advanced technique called Local Binary Pattern can also be used [3] to for palmprint feature extraction.

At the earlier stages researchers used only filters to extract features from palm. In [5] palmprint is considered as a piece of texture and 2-D Gabor filter is used to extract the features. This is called texture based feature extraction. The main disadvantage of this method is that filters cannot extract all the features.

The important aspect in multimodal biometric is the fusion or the combination of modalities. Many biometric traits including fingerprint and palmprint[17],face, palmprint and fingerprint[18], fingerprint, iris and face[19] have been used. But fusion of palmprint and fingerprint will give better result comparing to others. There are four levels of information fusion. Feature level fusion [17], score level fusion [32], pixel level fusion, and decision level fusion. Fusion increases accuracy, but it generally increases template sizes, computation costs and reduces user acceptance.

The task of matching is to calculate the degree of similarity between the input test image and a training image from database. Matching can be carried out in three ways: hierarchical approach [19], classification and coding. KNN classifier [17], nearest neighbour classifier (1-NN) based on the Euclidean space [31] are some of the commonly used classification approach.

Multimodal biometric systems have better accuracy and reliability. But sufficient attention has not been paid to security of multibiometric template. They are vulnerable to attacks including reply, database and brute-force attacks [23]. All the information that is generated by the scanners are stored as templates in the database. So leakage of biometric template information to unauthorized individuals constitutes a serious security issue. Therefore multibiometric template 
protection [22] should be carried out for security and privacy issues.

This paper mainly discusses the fusion of palmprint and fingerprint biometrics. However, the algorithm and analysis presented here can also be applied to other multimodal biometric fusion applications. Here Figure 1 illustrates a typical multimodal biometric authentication system. It consists of three main blocks that of pre-processing feature extraction and fusion. Bellow steps give a brief summary about the different steps involved in the proposed system.

1. Input image palmprint and finger print is given.

2. Selected features such as line, texture for palmprint and line and minutiae for fingerprint.

3. The features are merged by wavelet data fusion.

4. Support Vector Machine algorithm is used for classification of the image.

\section{PROPOSED DESIGN}

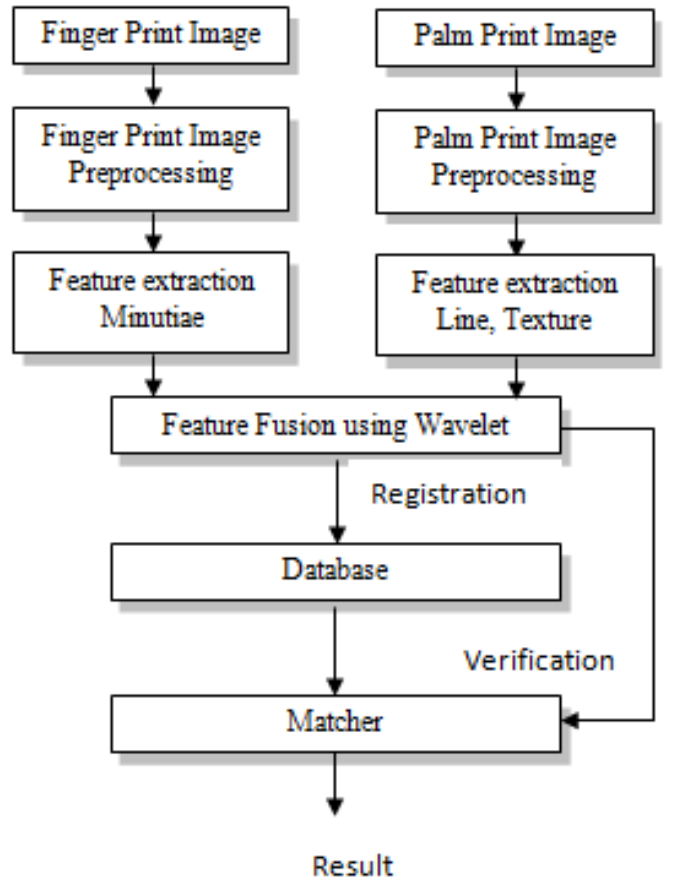

Figure 2. Proposed Multimodal Biometric System

Figure 2 shows the basic block diagram of the proposed method. Proposed system for multimodal biometric focuses on the feature level fusion. This methodology has the benefit of exploiting more amount of information from each biometric. Above figure comprises of pre-processing, feature extraction, fusion and matching. Pre-processing is used to remove the unwanted data in the input image. Feature extraction is done using Minutiae extraction for fingerprint and Local Binary Pattern for palmprint. Fusion is done using the Wavelet Fusion method. Fused images are stored in the database at the enrollment stage. Above steps are again done for palmprint and fingerprint images at the authentication stage. The features are then compared with the templates of the database to produce the output. Matching is done using the SVM. Combining more than one biometric system improves accuracy and reduces error rates. The proposed multimodal biometric system overcomes the limitations of individual biometric systems and also meets the accuracy requirements. Following sections will the details of the steps involved in the proposed system.

\subsection{Pre-processing}

The images must be pre-processed before going for next stage. Image pre-processing is done with the intention of removing unwanted data in the image such as noise, reflections etc.

All the images should have to be normalised in order to make it a single image size. This is the first stage of pre-processing.

Low pass filter is applied for both palmprint and fingerprint images to enhance the image. Electronic filter that pass low frequency signals but attenuates signals with frequency higher than the cut-off frequency is called low pass filter.

The next step is thresholding. Thresholding is used for binarization of images. Threshold $\mathrm{Tp}$ is used to convert

original image into binary image. Mathematically this transformation can be represented as

$$
\begin{array}{ll}
\mathrm{B}(\mathrm{x}, \mathrm{y})=1 & \text { if } \mathrm{O}(\mathrm{x}, \mathrm{y})^{*} \mathrm{~L}(\mathrm{x}, \mathrm{y})>=\mathrm{Tp} \\
\mathrm{B}(\mathrm{x}, \mathrm{y})=0 & \text { if } \mathrm{O}(\mathrm{x}, \mathrm{y})^{*} \mathrm{~L}(\mathrm{x}, \mathrm{y})<\mathrm{Tp}
\end{array}
$$

Where $\mathrm{B}(\mathrm{x}, \mathrm{y})$ and $\mathrm{O}(\mathrm{x}, \mathrm{y})$ are the binary images and the original image respectively. $\mathrm{L}(\mathrm{x}, \mathrm{y})$ represents a lowpass filter such as Gaussian and * represents an operator of convolution.

\subsection{Minutiae Extraction}

For extracting the features from the fingerprint image, minutiae extraction algorithm is used. The output of this algorithm will be the image template containing the minutiae details. There are two types of minutiae points. Ridge ending and Ridge bifurcation. A brief description of minutiae extraction algorithm is given bellow.

1) Consider the input image.

2) Scan the image from left to right, top to bottom order by following only ridges

3) Find the 0-1 transition, calculate the width of the ridge by noting the 1-0 transition.

4) Move to the next row and follow the same ridge. Note the width.

5) If the width $>=$ width in previous row there may be a top to bottom bifurcation. Then call the bifurcation function to check if it is a minutiae point.

Else

If the width $=<$ width in previous row there may be a bottom to top ridge bifurcation. Then call the bifurcation function to check if it is a minutiae point. 
6) Continue with the next row and repeat this for all the ridges in the given image or until 90 minutiae points have been obtained.

\subsection{Local Binary Pattern}

Unlike fingerprint which flows in uniform structure with alternating ridges and furrows, the texture of palmprint is irregular and the lines and ridges can flow in various directions. LBP is then used to analyse and describe the texture of the palmprint. The LBP operator is a simple but powerful texture descriptor that has been used in various applications. High discrimination ability and simplicity in computation have made it very suitable for palmprint feature extraction. LBP operator labels every pixel in an image by thresholding its neighboring pixels with the center value Bellow figure illustrates an example how the binary label for a pixel value is obtained.

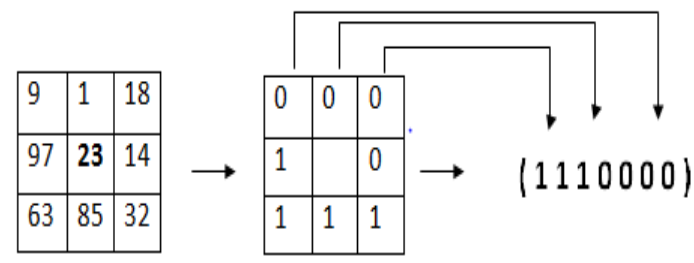

Figure 3. Local Binary Pattern

It is found that certain fundamental patterns in the bit string account for most of the information in the texture. These fundamental patterns are termed as "uniform" patterns and they are bit strings with at most 2 bitwise transitions from 0 to 1 and vice versa. Examples of uniform patterns include $00000000,11110000,00001100$. A label is given to each of the uniform patterns and the other "non-uniform" patterns are assigned to a single label. The next step is calculating the histogram of the labels.

\subsection{Wavelet fusion}

To fuse two images using wavelet fusion the two images should be of the same size and should be associated with the same color. Here we use wavelet data fusion to fuse the features of both palmprint and fingerprint.

\subsection{Support Vector Machine}

Support Vector Machine is a powerful learning tool based on statistical learning theory. An SVM is a binary classifier that makes its decision by constructing a linear decision boundary or hyper plane that optimally separate data points of the two classes in feature hyperspace [29] and also makes the margin maximized. SVMs have many advantages over Neural Networks. ANN are prone to the danger of over training [30] resulting in a solution over-fitted to the database being worked on. This could lead to overly optimistic results and accuracy outcomes. Secondly it has been found that SVMs are comparatively faster to train than ANNs.

\section{Results and Experimentations}

The effectiveness of our proposed multimodal biometric authentication scheme is evaluated on the palmprint and fingerprint database. The experiments are conducted in
MATLAB with image processing Toolbox and on a machine with an Intel core 2 Duo CPU Processor. Bellow figures shows some experimental results. Fig. 4(a) is the original input image. Binarization should have to be done before thinning. Fig. 4(c) shows the thinned image. Minutiae points are marked in 4(d). The two points, ridge ending and ridge bifurcations are differentiated with two colors. Fig. 4(e) is the original palmprint image. Region of Interest is taken from the palm image and that is given as input. Local Binary Pattern is used to extract the features. Fig $5(\mathrm{~g})$ explains the fused image using palmprint and fingerprint line image.

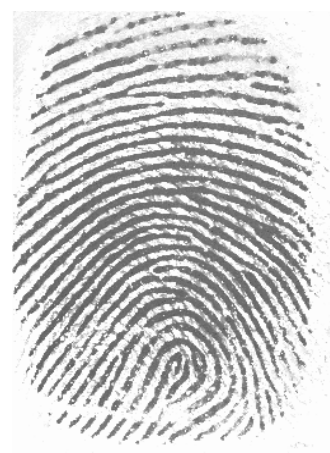

(a)

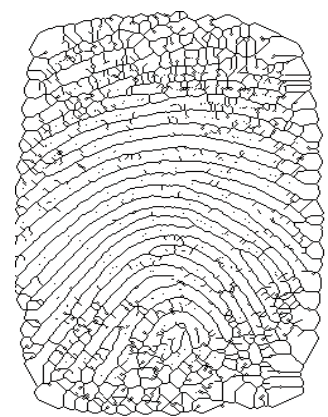

(c)

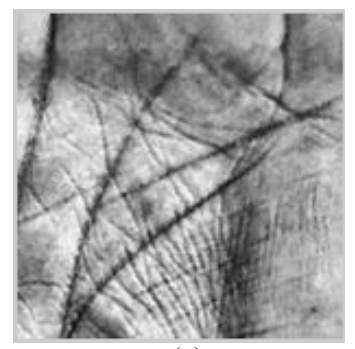

(e)

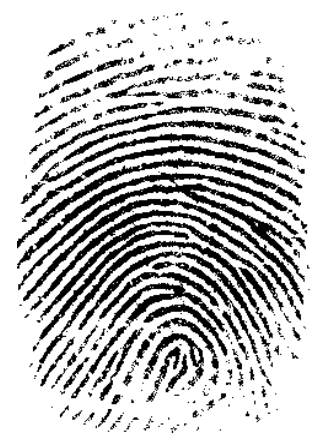

(b)

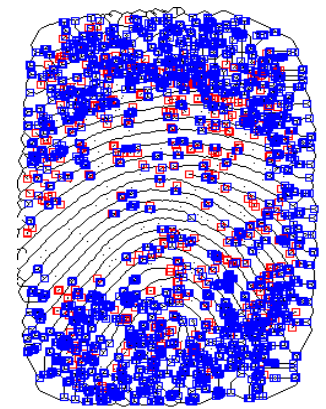

(d)

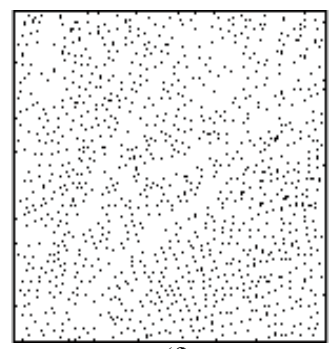

(f)
Figure 4. Feature Extraction : (a) Fingerprint image, (b) Binary image, (c) Thinned image, (d) Minutiae extraction, (e) Palmprint image, (f) Feature extraction using Local Binary Pattern. 


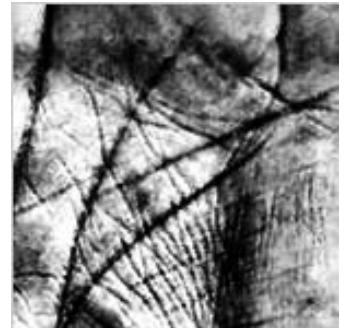

(a)

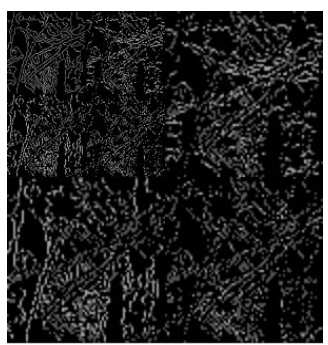

(c)

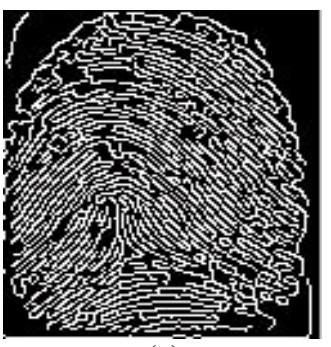

(e)

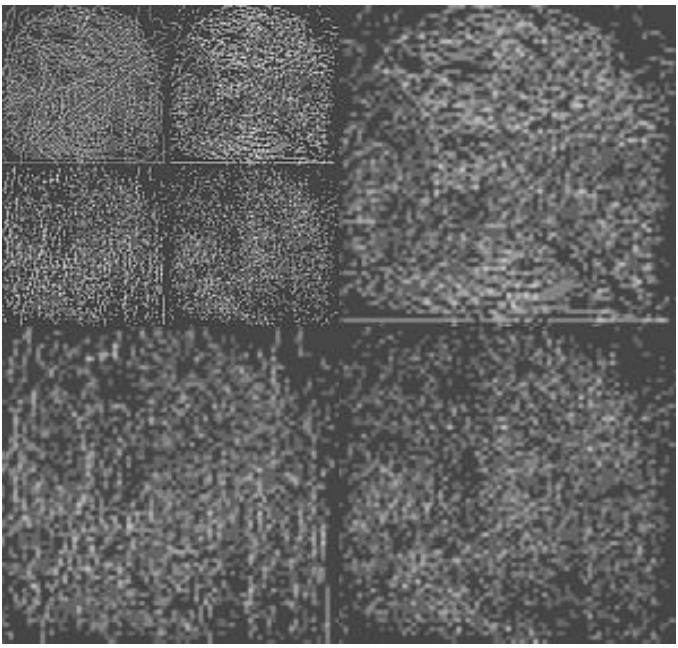

(g)

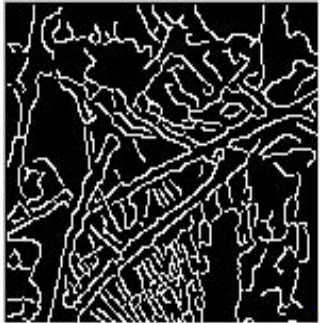

(b)

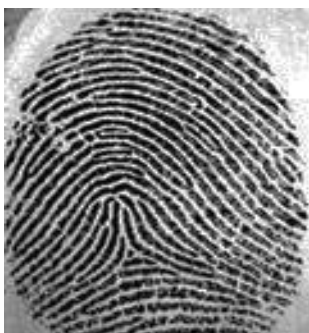

(d)

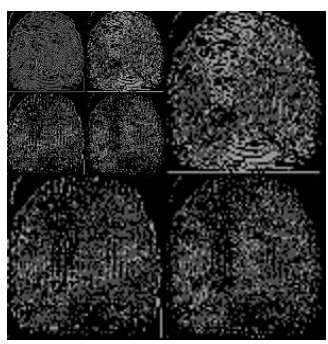

(f)

Figure 5. Feature Fusion : (a) Palmprint image, (b) Palmprint line detection, (c) Decomposition of palmprint image, (d) Fingerprint image, (e) Fingerprint line detection, (f) Decomposition of fingerprint image, (g) Fused image.

Fig. 6 shows the authentication part. Fig 6(a) shows the actual interface to select the images for the enrollment stage. After the enrollment we can choose images for autherisation(Fig 6(b)).

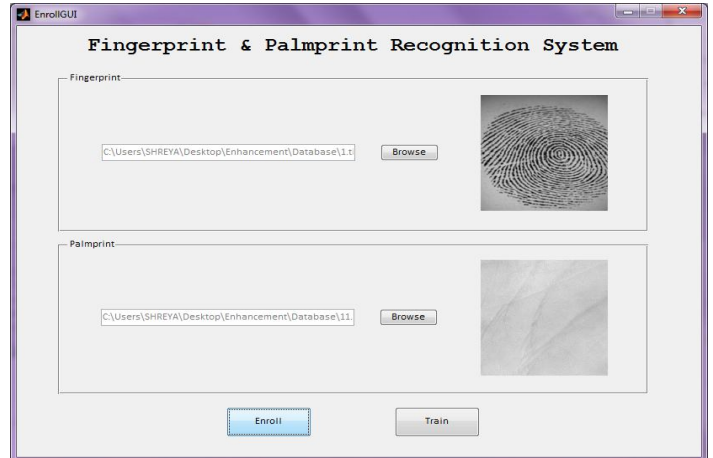

(a)

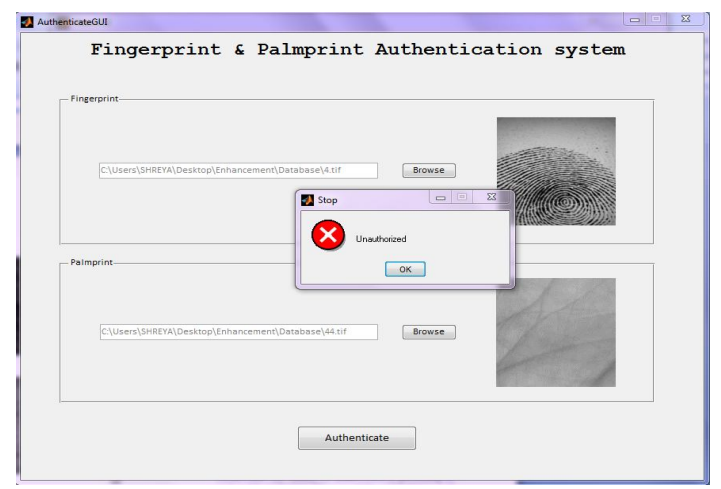

(b)

Figure 6. Authentication : (a) Enrollment stage, (b) Authentication stage.

\section{CONCLUSION}

We often face problems due to the fact that there exists only a single sample for personal identification in practice. The biometrics fusion technology is considered to be an effective solution to improve the performances of single sample biometrics systems. Meanwhile, the fusion of hand-based is promising in real world application because of the convenience and acceptance of the public. We have presented a feature level fusion scheme for palmprint and finger print verification and identification system using the combination of three palmprint representations. The extracted features from fingerprint and palmprint are fused using a wavelet based feature fusion technique. The combination of minutiae, line and Local Binary Pattern outperforms than using them individually.

\section{REFERENCES}

[1] D. Zhang, W.K. Kong, J. You, M. Wong. "On-line palmprint identification", In Proceedings of IEEE Transactions on Pattern Analysis and Machine Intelligence, 2003, 1041-1050.

[2] C.C. Han. "A hand-based personal authentication using a coarse-to-fine strategy", Image and Vision Computing, 2004, 909-918.

[3] Hanmandlu, M., Gureja and Jain, A. "Palm print recognition using Local Binary Pattern operator and support vector machines". Signal and Image Processing 
[4] (ICSIP), 2010 International Conference on (pp. 158162). IEEE.

[5] Deshpande, A., S., Patil, S., M., Lathi, R. "A Multimodel Biometric Recognition System based on Fusion of Palmprint Fingerprint and Face". International Journal of Electronics and Computer Science Engineering. ISSN2277-1956.

[6] Kong, W. K., Zhang, D., Li, W. "Palmprint feature extraction using 2-D Gabor filters". Pattern recognition, 36(10), 2003, 2339-2347.

[7] W. Li, D. Zhang, Z. Xu. "Palmprint identification by Fourier transform". International Journal of Pattern Recognition and Artificial Intelligence, 2002, 417-432.

[8] X. Wu, K. Wang, D. Zhang. "HMMs based palmprint identification". Lecture Notes in Computer Science. Springer, vol. 3072, 2004. pp. 775-781.

[9] Han, C., C. "A hand-based personal authentication using a coarse-to-fine strategy". Image and Vision Computing 22 (11), 2004, 909-918.

[10] Han, C. C., Cheng, H. L., Lin, C. L., Fan, K. C. "Personal authentication using palm-print features". Pattern Recognition, 36(2),2003, 371-381.

[11] Kumar, A., Zhang, D. "Integrating shape and texture for hand verification". International Journal of Image and Graphics, 6(01), 2006,101-113.

[12] Poon, C., Wong, D. C. M., \& Shen, H. C. "A new method in locating and segmenting palmprint into region-of-interest". In Pattern Recognition, 2004. ICPR 2004. Proceedings of the 17 th International Conference on (Vol. 4, pp. 533-536). IEEE.

[13] Wu, X., Wang, K., Zhang, D. "Line feature extraction and matching in palmprint". In Society of Photo-Optical Instrumentation Engineers (SPIE) Conference Series (Vol. 4875, pp. 583-590).

[14] Han, C. C., Cheng, H. L., Lin, C. L., \& Fan, K. C. "Personal authentication using palm-print features". Pattern Recognition, 2003, 36(2), 371-381.

[15] You, J., Kong, W. K., Zhang, D., \& Cheung, K. H. “On hierarchical palmprint coding with multiple features for personal identification in large databases". Circuits and Systems for Video Technology, IEEE Transactions on, 14(2), 2004, 234-243.

[16] Pang, Y. H., Connie, T., Jin, A., \& Ling, D. "Palmprint authentication with Zernike moment invariants". In Signal Processing and Information Technology, 2003. ISSPIT 2003. Proceedings of the 3rd IEEE International Symposium on (pp. 199-202). IEEE.

[17] Albert, T., A. Ganesan, S. “Application of Principal Component Analysis in Multimodel Biometric Fusion System". European Journal of scientific research, 2012, Vol 67, No. 2.

[18] Gayathri, R. Ramamoorthy, P. "Fingerprint and palmprint Recognition Approach based on Multiple Feature extraction". European Journal of scientific research, 2012, Vol 76, No 4.

[19] Deshpande, A., S., Patil, S., M., Lathi, R. “A Multimodel Biometric Recognition System based on Fusion of Palmprint Fingerprint and Face". International Journal of
Electronics and Computer Science Engineering, 2012, ISSN-2277-1956.

[20] J. You, W.K. Kong, D. Zhang, K.H. Cheung, "On hierarchical palmprint coding with multiple features for personal identification in large databases", IEEE Transactions on Circuits and Systems for Video Technology 14 (2) ,2004, 234-243.

[21] Joshitha N., J. Medona S., R. "Image Fusion using PCA in Mutifeature Based Palmprint Recognition". International Journal of soft computing and Engineering, 2012, ISSN:2231-2307,Volume-2,Issue-2.

[22] Xu, Y., Zhang, D., \& Yang, J. Y. "A feature extraction method for use with bimodal biometrics". Pattern recognition, 43(3), 2010, 1106-1115.

[23] Nagar, A., Nandakumar, K., \& Jain, A. K. "Multibiometric Cryptosystems Based on Feature-Level Fusion". Information Forensics and Security, IEEE Transactions on, 7(1), 2012, 255-268.

[24] Ratha, N. K., Connell, J. H., Bolle, R. M. "Biometrics break-ins and band-aids". Pattern Recognition Letters, 24(13), 2003, 2105-2113.

[25] Jain, A. K., Prabhakar, S., Hong, L. "A multichannel approach to fingerprint classification". Pattern Analysis and Machine Intelligence, IEEE Transactions on, 21(4), 1999, 348-359.

[26] Cui, J., Xu, Y. "Three dimensional palmprint recognition using linear discriminant analysis method". In Innovations in Bio-inspired Computing and Applications (IBICA), 2011 Second International Conference on. IEEE.

[27] Zhao, F., Tang, X. "Preprocessing and post-processing for skeleton-based fingerprint minutiae extraction". Pattern Recognition, 40(4), 2007, 1270-1281.

[28] Zhao, F., Tang, X. "Preprocessing and post processing for skeleton-based fingerprint minutiae extraction". Pattern Recognition 40, 2007, 1270 - 1281.

[29] Zhao, F., Tang, X. "Preprocessing and post processing for skeleton-based fingerprint minutiae extraction". Pattern Recognition 40, 2007, 1270 - 1281.

[30] Vapnik, V. "The nature of statistical learning theory". Springer-Verlag, Berlin, 1995.

[31] Bernhard Schölkopf and Alex Smola, "Learning with Kernels" (MIT Press, Cambridge, MA, 2002).

[32] Zhang, Y., Sun, D., Qiu, Z. "Hand-based feature level fusion for single sample biometrics recognition". In Emerging Techniques and Challenges for Hand-Based Biometrics (ETCHB), 2010 International Workshop on (pp. 1-4). IEEE.

[33] Kumar, A., Zhang, D. "Combining fingerprint, palmprint and hand-shape for user authentication". In Pattern Recognition, 2006. ICPR 2006. 18th International Conference on (Vol. 4, pp. 549-552). IEEE. 